\title{
NUMERICAL EFFECTIVENESS OF DIFFERENT FORMULATIONS OF THE RIGID FINITE ELEMENT METHOD
}

\author{
I. ADAMIEC-WÓJCIK*, Ł. DRĄG and S. WOJCIECH \\ Division of Transport and Computer Sciences \\ Faculty of Management and Transport \\ University of Bielsko-Biała \\ Willowa str. 2, 43-309 Bielsko-Biała, POLAND \\ E-mails: i.adamiec@ath.bielsko.pl; ldrag@ath.bielsko.pl; swojciech@ath.bielsko.pl
}

\begin{abstract}
The paper presents an application of different formulations of the rigid finite element method (RFEM) to dynamic analysis of flexible beams. We discuss numerical effectiveness of the classical RFEM and an alternative approach in which continuity of displacements is preserved by means of constraint equations. The analysis is carried out for a benchmark problem of the spin-up motion in planar and spatial cases. Torsion is omitted for numerical simulations and two cases of the new approach are considered. The results obtained by means of these methods are compared with the results obtained using a nonlinear two-node superelement.
\end{abstract}

Key words: flexibility of links, rigid finite element method, dynamic analysis, nonlinear motion, numerical effectiveness.

\section{Introduction}

Modeling dynamics of manipulators with flexible links remains an open field of research. Although researchers has been involved in this field for years (Dwivedy and Eberhard, 2006), there is still a need for numerically efficient models which account for nonlinear phenomena occurring during dynamic analysis of mechanism with highly flexible links. The rigid finite element method (RFEM) formulated by Kruszewski et al. (1975) has been widely used and developed so that new formulations of the method can be characterized either by new features like the modified formulation (MRFEM) presented by Adamiec-Wójcik and Wojciech (1993) or by a different notation which uses homogenous transformations (Wittbrodt et al., 2006).

This paper uses different formulations of the RFEM. A systematic approach by means of homogenous transformations is used for derivations of the equations of motion. However, since the numerical effectiveness is our aim, the resulting algorithms use explicit expressions of matrix and vector elements. Apart from the classical formulation, a new approach in which the rigid finite elements are connected by means of constraint equations is used. This new formulation was applied to modelling planar flexible ropes by Adamiec-Wójcik et al. (2012). The same approach was used in modelling risers in the paper (Adamiec-Wójcik et al., 2013) where additionally the influence of water environment has been taken into account. For the purpose of this paper the spatial model is considered and it will be shown that despite the necessity of solving constraint equations the method is very efficient and times of calculations are shorter than those for other approaches.

Simulations are carried out for a benchmark problem of a spinning beam with a spin-up movement and similar movement but with simultaneously excited vibrations in the out-of-plane direction. The results are compared with those presented by Boer et al. (2014) and the comparisons show very good agreement.

\footnotetext{
* To whom correspondence should be addressed
} 


\section{Models of a flexible link}

Discretization by means of the rigid finite element method is carried out in two steps called primary and secondary divisions. First, a continuous beam-like flexible link with constant cross-section is divided into $n$ equal elements and then spring-damping elements reflecting flexible features are placed in the middle of segments obtained during the first division. Finally, a system of $n+1$ rigid elements (rfe) connected by means of $n$ spring-damping elements (sde) is obtained. The approaches described below differ in the manner of assigning the local coordinate systems and the choice of generalized coordinates describing the motion of elements.

\subsection{Classical rigid finite element method}

Local coordinate systems $\{i\}^{\prime}$ are placed in the center of mass of the element (Fig.1) and the axes of the system coincide with the principal central axes of the element.

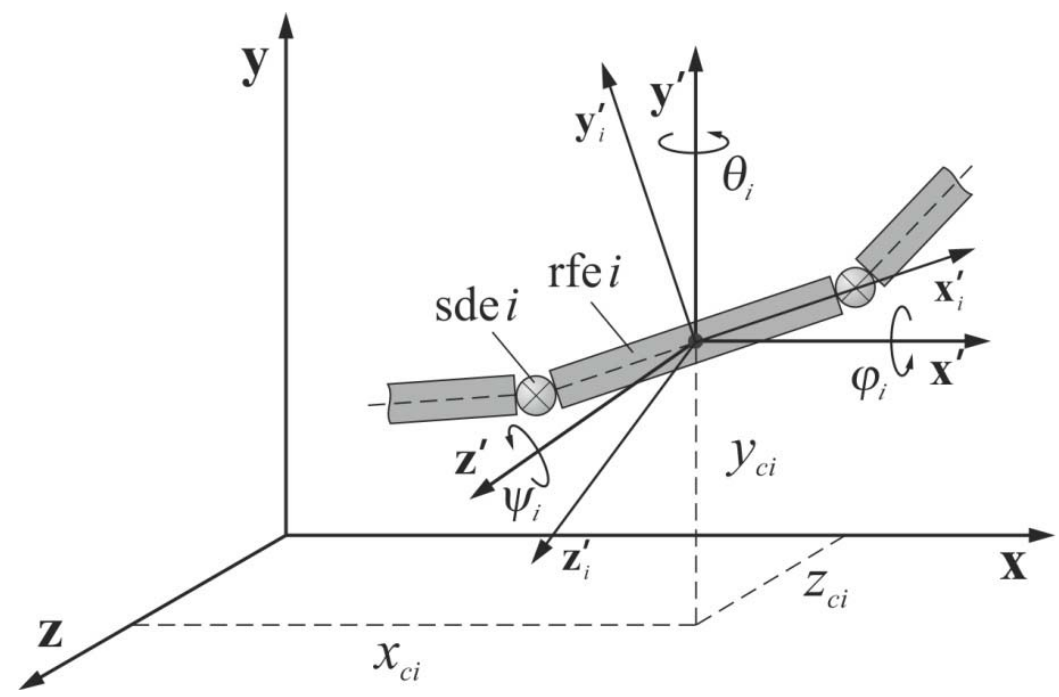

Fig.1. Local coordinate systems and generalized coordinates (classical RFEM).

The position of the element is described with respect to the inertial coordinate system by six generalized coordinates which are the elements of the following vector

$$
\boldsymbol{q}_{i}^{S}=\left[\begin{array}{llllll}
x_{C i} & y_{C i} & z_{C i} & \psi_{i} & \theta_{i} & \phi_{i}
\end{array}\right]^{T} .
$$

When large deflections of a flexible link are considered, trigonometrical functions of angles $\psi_{i}, \theta_{i}, \phi_{i}$ cannot be linearized. Since the axes of the local coordinate system of rfe $i$ are chosen to coincide with the principal central axes of inertia the mass features of the element are described by the elements of the following vector

$$
\boldsymbol{H}_{i}=\left[\begin{array}{llll}
h_{x}^{(i)} & h_{y}^{(i)} & h_{z}^{(i)} & m_{i}
\end{array}\right]^{T}
$$

where

$$
h_{x}^{(i)}=\int_{m_{i}} x_{i}^{\prime 2} d m ; \quad h_{y}^{(i)}=\int_{m_{i}} y_{i}^{\prime 2} d m ; \quad h_{z}^{(i)}=\int_{m_{i}} z_{i}^{\prime 2} d m .
$$


The equations of motion of the flexible link are derived from the Lagrange equations and following the procedure described in Wittbrodt et al. (2006) the Lagrange operators can be written as

$$
\boldsymbol{\varepsilon}_{i}\left(E_{i}\right)=\frac{d}{d t} \frac{\partial E_{i}}{\partial \dot{\boldsymbol{q}}_{i}^{s}}-\frac{\partial E_{i}}{\partial \boldsymbol{q}_{i}^{s}}=\boldsymbol{M}_{i}^{s} \ddot{\boldsymbol{q}}_{i}^{s}+\boldsymbol{h}_{i}^{s}
$$

where

$$
\boldsymbol{M}_{i}^{s}=\boldsymbol{M}_{i}^{s}\left(\psi_{i}, \theta_{i}, \phi_{i}\right), \quad \boldsymbol{h}_{i}^{s}=\boldsymbol{h}_{i}^{s}\left(\psi_{i}, \theta_{i}, \phi_{i}, \dot{\psi}_{i}, \dot{\theta}_{i}, \dot{\phi}_{i}\right)
$$

The choice of the generalized coordinates as absolute angles is an advantage when deriving the parts of the equations of motion which result from the kinetic energy, yet, the calculations of expressions resulting from the energy of spring deformations are more complicated (Wittbrodt et al., 2013). The continuity of displacements is not ensured and the relation among rfes is described by sdes. Finally, the equations of motion take the following form

$$
\boldsymbol{M}^{s} \ddot{\boldsymbol{q}}^{s}=\boldsymbol{Q}^{s}+\boldsymbol{h}^{s}-\frac{\partial V^{s}}{\partial \boldsymbol{q}^{s}}
$$

where $\boldsymbol{q}^{s}=\left[\begin{array}{lll}\boldsymbol{q}_{0}^{s T} & \cdots & \boldsymbol{q}_{n}^{s T}\end{array}\right]^{T}, \boldsymbol{M}^{s}$ is a block-diagonal mass matrix.

The equations of motion in this formulation have to be integrated using a small integration step due to large translational stiffness (shear and longitudinal) which results in high frequency vibrations.

\subsection{An alternative formulation}

In this approach shear stiffness is eliminated. In order to consider longitudinal flexibility an additional spring-damping element responsible for elongation is placed in the middle of each rfe (Fig.2).

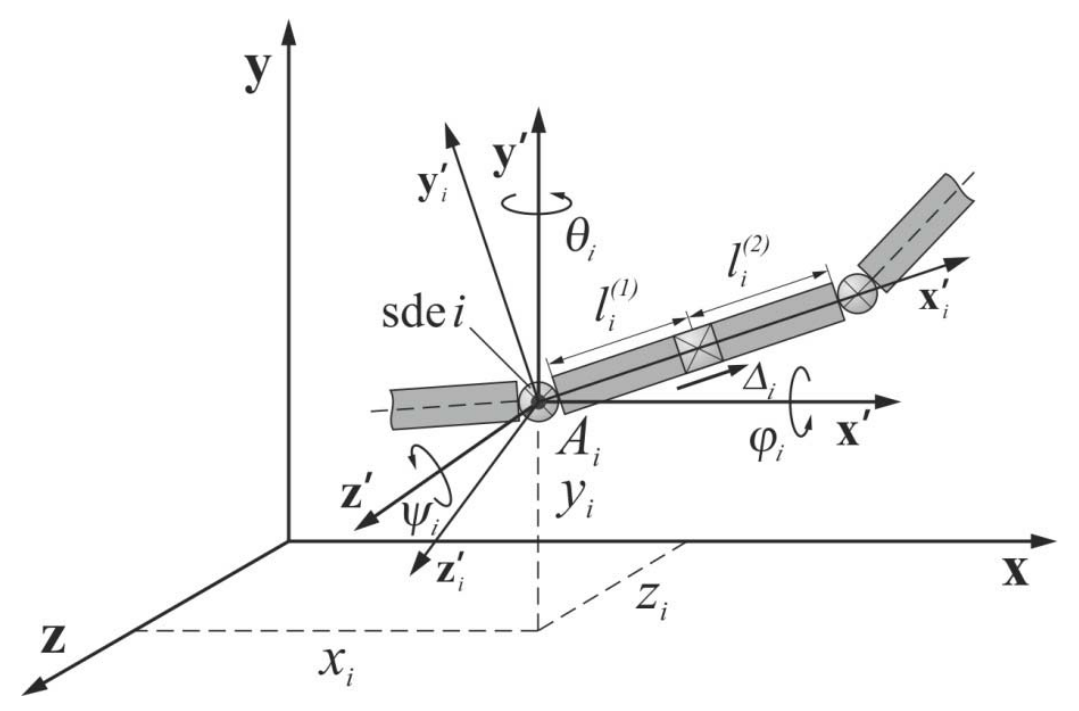

Fig.2. Local coordinate systems and generalized coordinates (alternative approach).

The local coordinate system is placed at the preceding sde. The vector of generalized coordinates is defined as follows 


$$
\boldsymbol{q}_{i}^{l}=\left[\begin{array}{lllllll}
x_{i} & y_{i} & z_{i} & \psi_{i} & \theta_{i} & \phi_{i} & \Delta_{i}
\end{array}\right]^{T}
$$

Having taken into account the different definition of translational displacements $\left(x_{i}, y_{i}, z_{i}\right.$ are coordinates of point $A_{i}$, not the center of the mass), the kinetic energy of the element can be calculated as

$$
E_{i}=E_{i}^{(1)}+E_{i}^{(2)}
$$

where

$$
E_{i}^{(l)}=E_{i}^{(l)}\left(x_{i}, y_{i}, z_{i}, \psi_{i}, \theta_{i}, \phi_{i}\right) ; \quad E_{i}^{(2)}=E_{i}^{(2)}\left(x_{i}, y_{i}, z_{i}, \psi_{i}, \theta_{i}, \phi_{i}, \Delta_{i}\right)
$$

The Lagrange operators defined in Eq.(2.3) take the form

$$
\boldsymbol{\varepsilon}_{i}\left(E_{i}\right)=\boldsymbol{M}_{i}^{l} \ddot{\boldsymbol{q}}_{i}^{l}+\boldsymbol{h}_{i}^{l}
$$

where $\boldsymbol{M}_{i}^{l}$ is matrix $7 \times 7$ with elements dependent on $\boldsymbol{q}_{i}^{l}, \boldsymbol{h}_{i}^{l}$ is a vector with 7 elements dependent on $\boldsymbol{q}_{i}^{l}$ and $\dot{\boldsymbol{q}}_{i}^{l}$.

In order to calculate forces and moments resulting from sdes, only forces of longitudinal deformations (dependent on $\Delta_{i}$ ) and moments dependent on subtractions of angles $\psi_{i}, \theta_{i}, \phi_{i}$ and $\psi_{i-1}, \theta_{i-1}, \phi_{i-1}$ or $\psi_{i+1}, \theta_{i+1}, \phi_{i+1}$ are taken into account. The continuity of displacements at point $A_{i}$ is ensured by means of constraints equations which can be written in the form

$$
\boldsymbol{r}_{A_{i}}^{(i-1)}\left(\boldsymbol{q}_{i-1}^{l}\right)=\boldsymbol{r}_{A_{i}}^{(i)}
$$

where $\quad \boldsymbol{r}_{A_{i}}^{(i)}=\left[\begin{array}{lll}x_{i} & y_{i} & z_{i}\end{array}\right]^{T}$

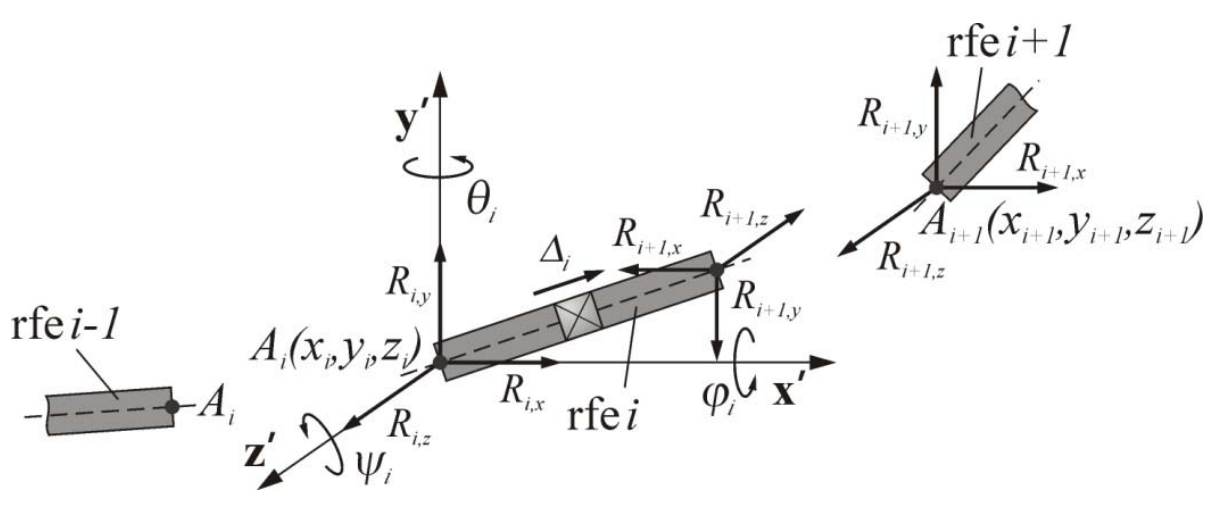

Fig.3. Constraint reactions.

In this formulation constraint reactions (Fig.3) have to be introduced in the equations of motion and the resulting generalized forces can be written in the following form

$$
\boldsymbol{Q}_{i}\left(\boldsymbol{R}_{i}, \boldsymbol{R}_{i+1}\right)=\overline{\mathbf{D}} \boldsymbol{R}_{i}+\overline{\boldsymbol{D}}_{i} \boldsymbol{R}_{i+1}
$$


where $\quad \boldsymbol{R}_{i}=\left[\begin{array}{lll}R_{i, x} & R_{i, y} & R_{i, z}\end{array}\right]^{T}, \quad \overline{\boldsymbol{D}}_{i}=\overline{\boldsymbol{D}}_{i}\left(\boldsymbol{q}_{i}^{l}\right)$.

By double differentiation of the constraint equations the final form of the equations of motion (Adamiec-Wójcik and Brzozowska, 2013) can be obtained

$$
\begin{aligned}
& \boldsymbol{M}^{i} \ddot{\boldsymbol{q}}^{l}-\boldsymbol{D} \boldsymbol{R}=\boldsymbol{Q}^{l}-\boldsymbol{h}^{l}-\frac{\partial V^{l}}{\partial \boldsymbol{q}^{l}}, \\
& \boldsymbol{D}^{T} \ddot{\boldsymbol{q}}=\boldsymbol{G} .
\end{aligned}
$$

The Baumgart method is used for stabilisation of constraint equations. Due to a special structure of the matrix of constraint reactions and block-diagonal mass matrix, the problem can be replaced by the solution of reactions from a system of $3(n+1)$ linear algebraic equations with a band of less than 22 elements. Some additional multiplications of vectors and matrices enable us to calculate the acceleration vector at each integration step.

In the special case when longitudinal deformations can be neglected, vectors $\boldsymbol{q}^{l}-\boldsymbol{q}_{i}^{l}$ have only six components. Thus all large values of translational stiffness can be omitted. In such a case the formulation is like the modification of the rigid finite element method presented in Adamiec-Wójcik and Wojciech (1993) but without its essential drawback which is the full mass matrix.

\section{Numerical simulations}

In this section we consider two benchmark problems for examining numerical effectiveness of the different formulations of the Rigid Finite Element Method. The results obtained are also compared with those presented by Boer et al. (2014). The first example is a spinning planar beam very often used in literature for analysis of highly nonlinear motions. The second example allows us to consider full threedimensional motion and it is a spinning beam attached to a universal joint by means of which out-of-plane vibrations are excited.

In the simulations presented below torsion is omitted and the beam is discretized by means of the three formulations of the Rigid Finite Element Method denoted as follows:

$\mathrm{S}$ - the model by the classical rigid finite element method described in section 2.1; the vector of generalized coordinates is $\boldsymbol{q}_{i}^{s}=\left[\begin{array}{llllll}x_{C i} & y_{C i} & z_{C i} & \psi_{i} & \phi_{i}\end{array}\right]^{T}$; three translational stiffness of sde: two shear and one longitudinal are considered;

$\mathrm{L}$ - the model by the new approach with the additional spring element described in section 2.2; the vector of generalized coordinates is $\boldsymbol{q}_{i}^{l}=\left[\begin{array}{lllllll}x_{i} & y_{i} & z_{i} & \psi_{i} & \phi_{i} & \Delta_{i}\end{array}\right]^{T}$; shear stiffness is omitted but longitudinal one is taken into account;

B - the model by the new approach but without longitudinal flexibility; the vector of generalized coordinates is $\boldsymbol{q}_{i}^{b}=\left[\begin{array}{lllll}x_{i} & y_{i} & z_{i} & \psi_{i} & \phi_{i}\end{array}\right]^{T}$; all translational stiffness are omitted.

\subsection{Spinning beam}

The flexible beam (Fig.4) with length $l=8 \mathrm{~m}$, a constant rectangular cross-section $a x b, a=0.03675$ $m, b=0.001986 \mathrm{~m}$ is considered. Material parameters of the beam are those of aluminum: Young modulus $E=6.895 \cdot 10^{10} \mathrm{~N} / \mathrm{m}^{2}$, density $\rho=2766 \mathrm{~kg} / \mathrm{m}^{3}$, Poisson number $\nu=0.3$. 


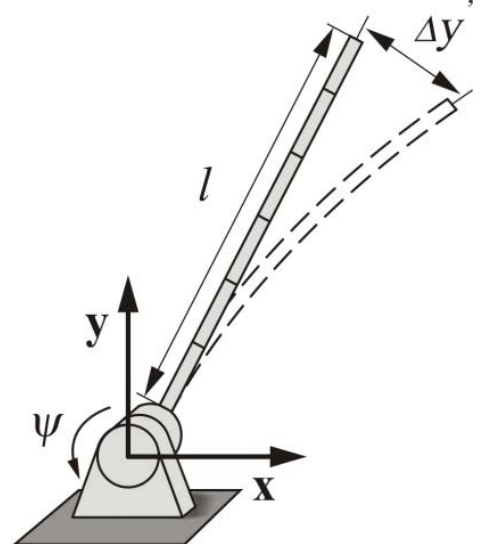

Fig.4. Model of a spinning beam.

The motion of the beam is defined by the change of angle $\psi$ by the following formula

$$
\Psi(t)= \begin{cases}\frac{\Omega}{T}\left[\frac{1}{2} t^{2}-\frac{T^{2}}{4 \pi^{2}}\left(1-\cos \frac{2 \pi t}{T}\right)\right], & 0 \leq t \leq T \\ \Omega\left(t-\frac{1}{2} T\right), & t>T\end{cases}
$$

where $\Omega=4 \mathrm{rad} / \mathrm{s}$ is the final angular velocity and $T=15 \mathrm{~s}$ is the spin-up time.

The change of the angle $\psi$ and velocity in time are presented in Fig.5
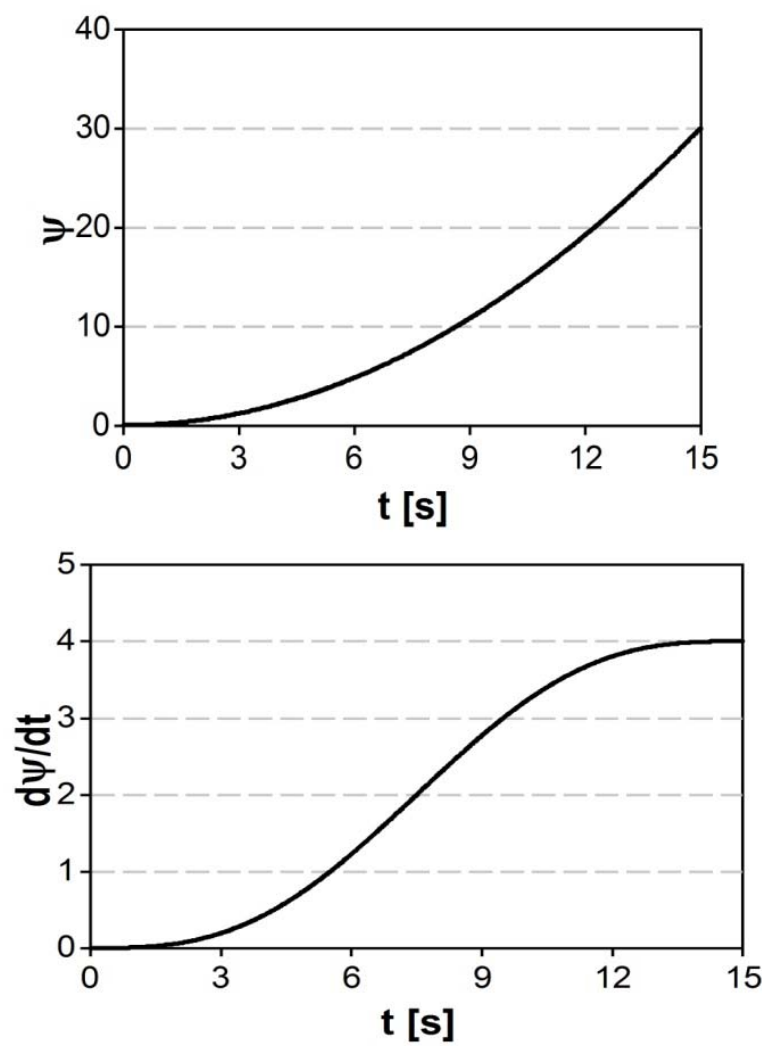

Fig.5. Spin-up motion. 
In order to check the correctness of the models, the results are compared with those presented by Boer et al. (2014) denoted as A (Fig.6).

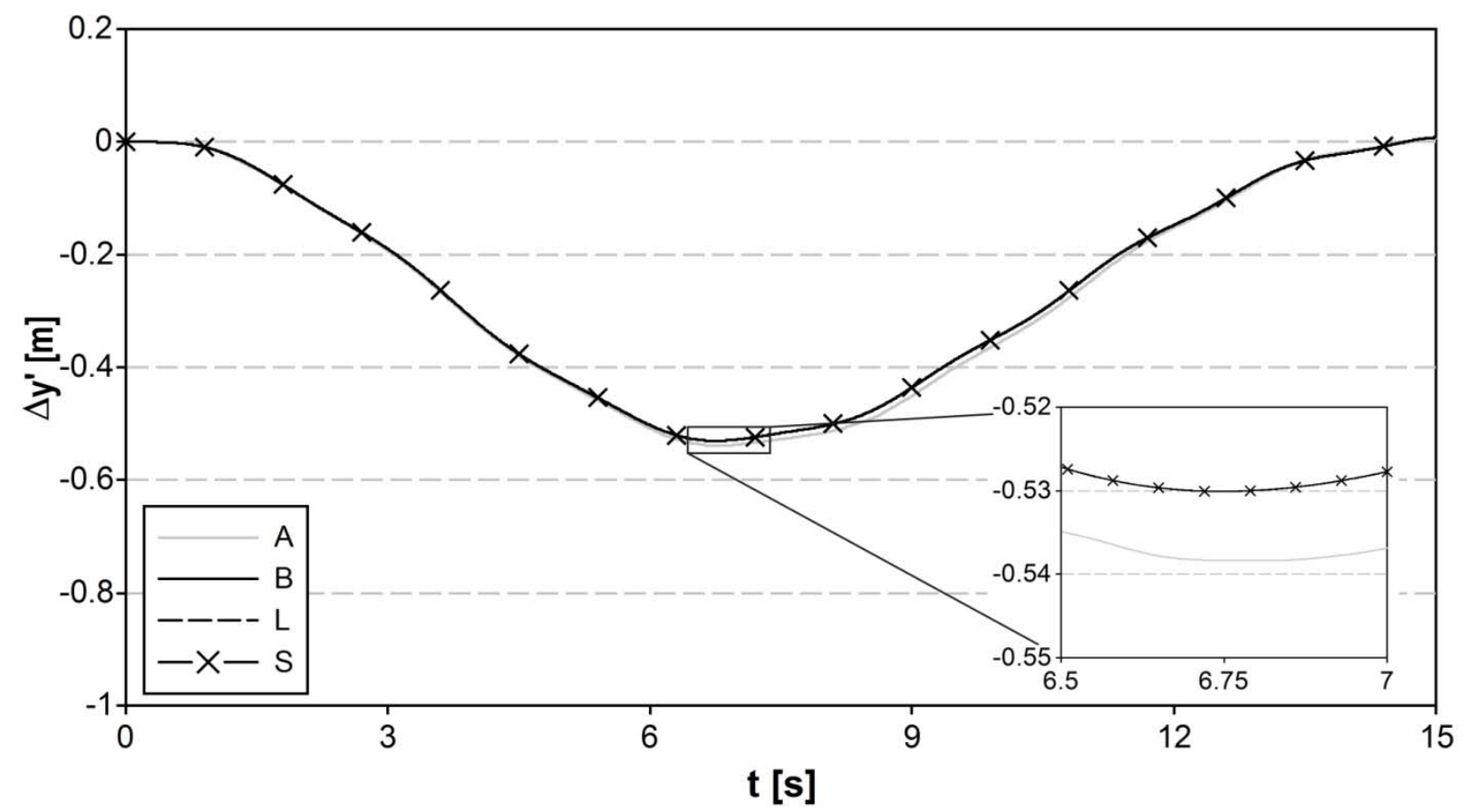

Fig.6. Course of the beam tip deflection.

It can be seen that the results obtained are in very good agreement with those presented in the mentioned paper, where the authors proposed a two-node super-element for modeling flexibility. They compared their results with a model using nonlinear planar beam elements with elongation modes suppressed.

Figure 7 shows a comparison of calculation times for a different number of rigid elements into which the beam is discretized.

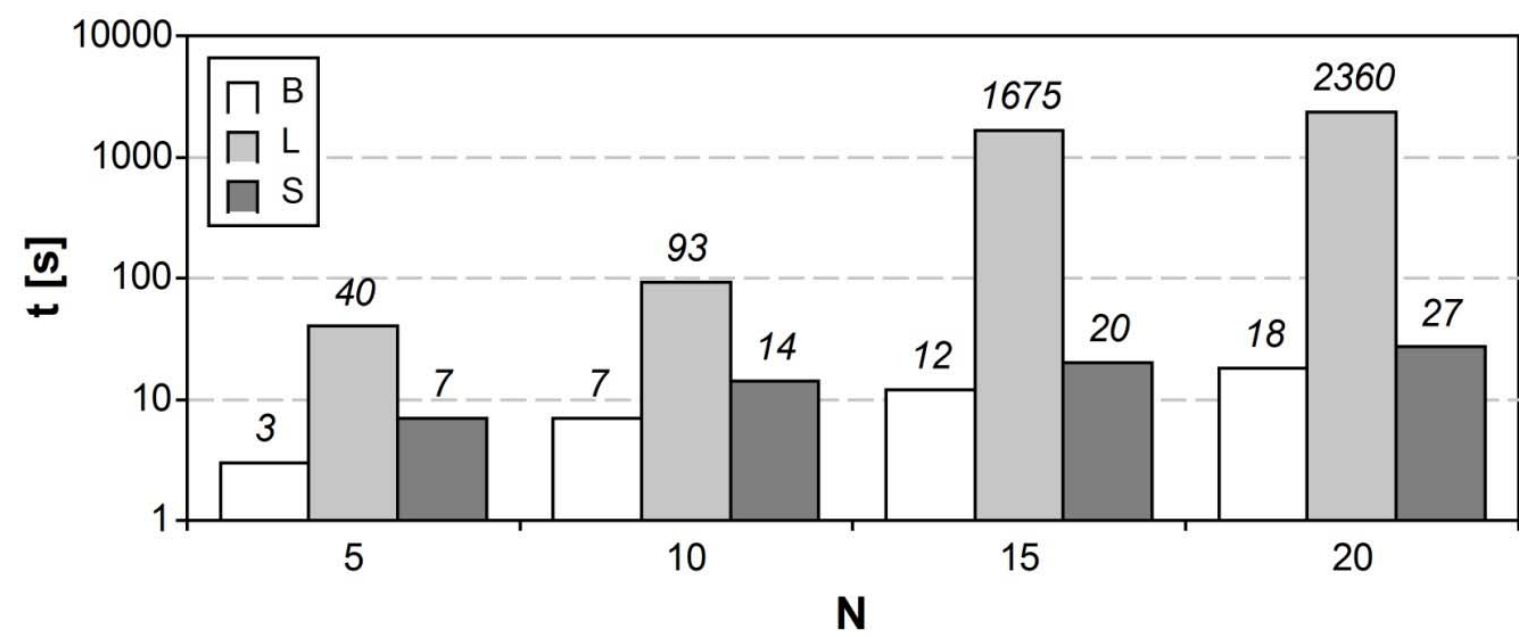

Fig.7. Comparison of calculation times for a different number of rfes. 
The shortest calculation time is obtained for the new approach of the RFEM when the elongation is omitted, which is due to elimination of vibrations with high free frequencies.

\subsection{Spinning beam with out-of-plane vibrations}

The beam considered for the analysis of the spatial motion (Fig.8) has the same material properties as in the first examples. It has the same length of $8 \mathrm{~m}$, but the constant cross-section is assumed as $w \mathrm{x} h$, $w=0.02 \mathrm{~m}, h=0.04 \mathrm{~m}$.

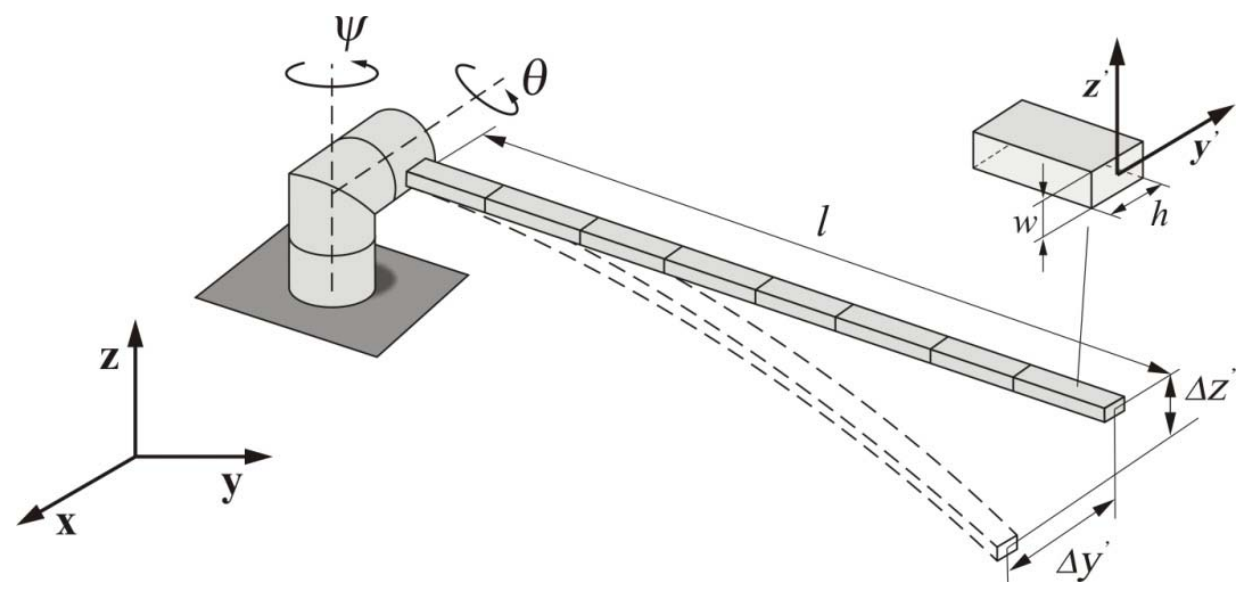

Fig.8. Model of the spatial beam.

The beam undergoes spin-up motion about $\boldsymbol{z}^{\prime}$ axis defined by the change of angle $\psi$ (3.1) and additional excitations about $\boldsymbol{y}^{\prime}$ axis are defined by the formula

$$
\theta=0.01 \sin (15 t) \text {. }
$$

The character of changes can be seen in Fig.9.

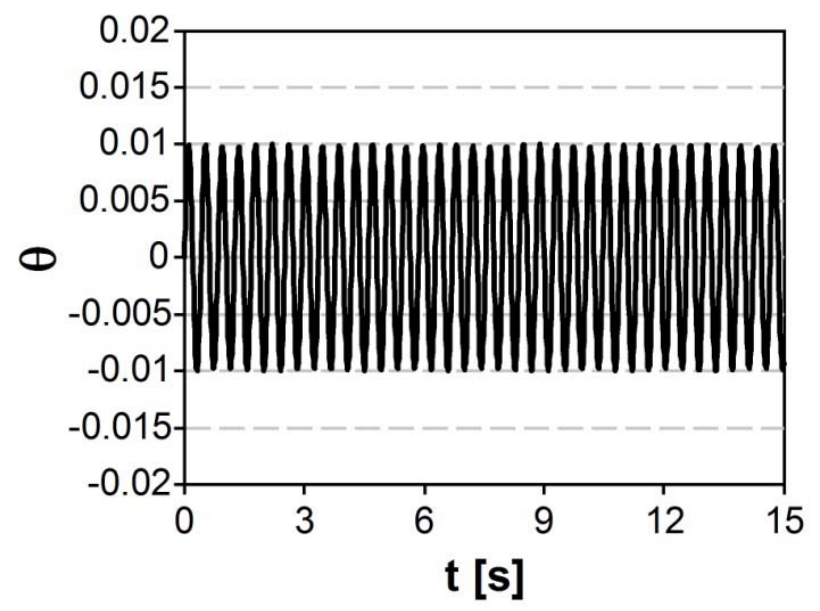

Fig.9. Course of out-of plane motion. 
Figures 10 and 11 show the tip deflections $\Delta y^{\prime}$ and $\Delta z^{\prime}$ in horizontal and vertical directions. The results obtained with our models are compared with those presented in Boer et al. (2014) denoted as A.

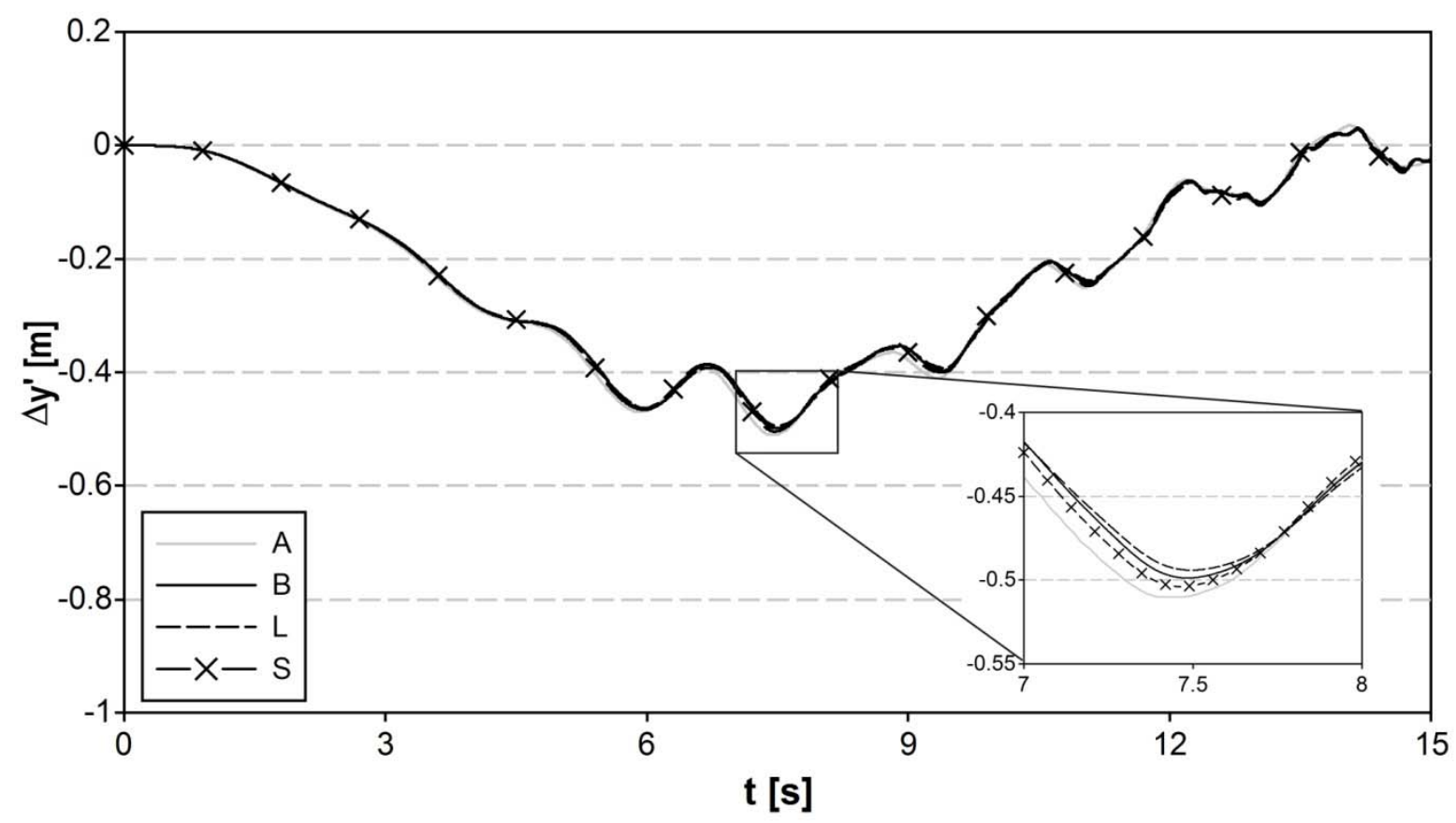

Fig.10. Tip deflection $\Delta y^{\prime}$.

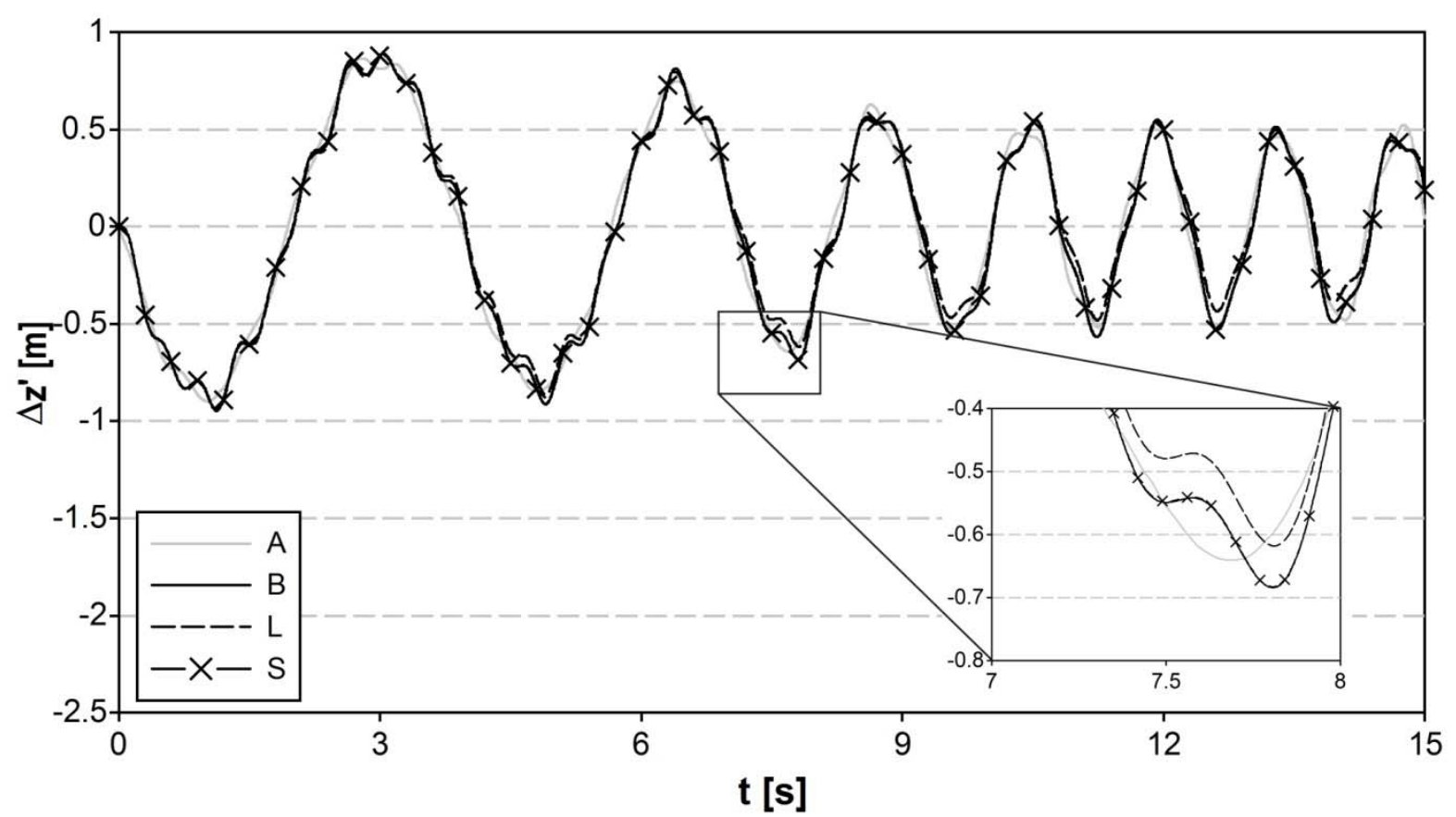

Fig.11. Tip deflection $\Delta z^{\prime}$.

The results obtained by the rigid finite element method (in all formulations) are in good agreement with those obtained by means of a different method. 
Figure 12 presents a comparison of time of calculations for a different number of elements from discretization.

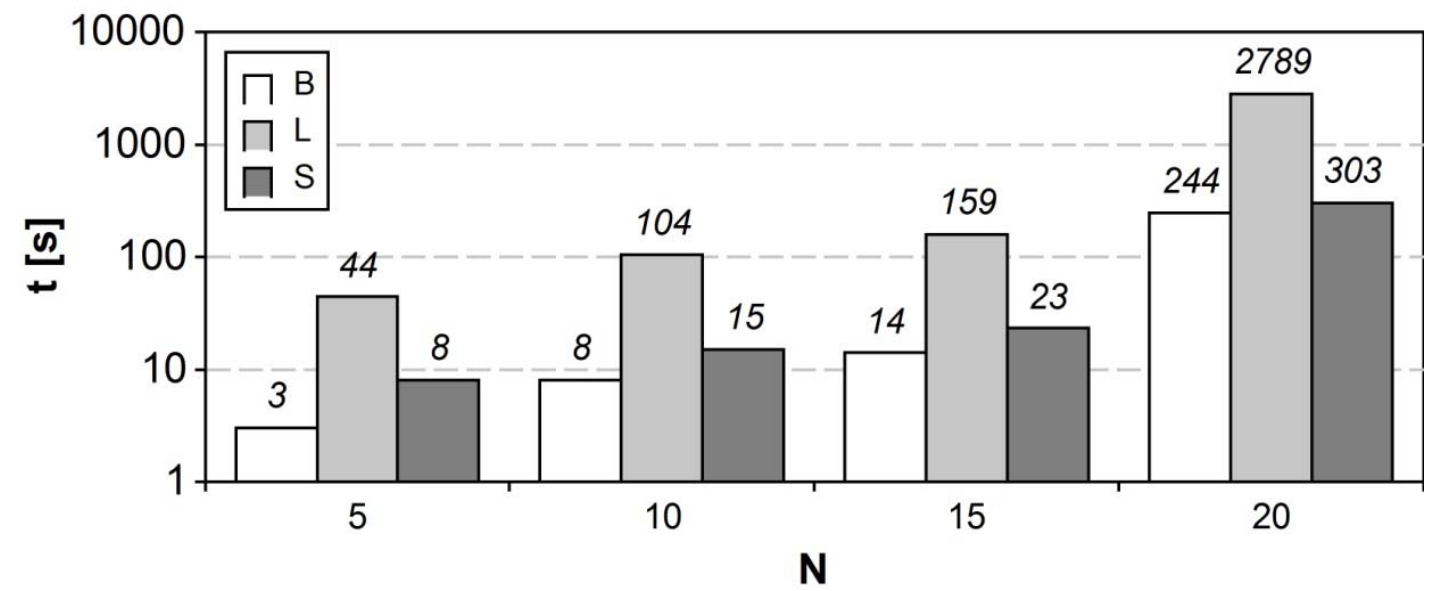

Fig.12. Comparison of calculation time.

It can be observed that consideration of longitudinal flexibility increases the calculation time significantly. When the elongation is omitted, the new approach allows us to reduce almost by half the calculation time with comparison to the classical RFEM. This is due to elimination of the coefficients of the translational stiffness which are large and thus frequencies of free vibrations are low. This directly influences the length of the integration step for the equations of motion. It can be 10 times larger in case B than in cases $\mathrm{S}$ and $\mathrm{L}$.

\section{Final remarks}

The paper presents different formulations of the rigid finite element method with the emphasis on their numerical effectiveness. All approaches presented are characterized by similar exactness and the error with respect to the nonlinear finite element method is insignificant. Calculation time is shortest for the alternative approach when the elongation is omitted. The method presented in the paper can be especially effective in modeling of cables, ropes and risers and due to its numerical effectiveness can be used in problems connected with control.

\section{Nomenclature}

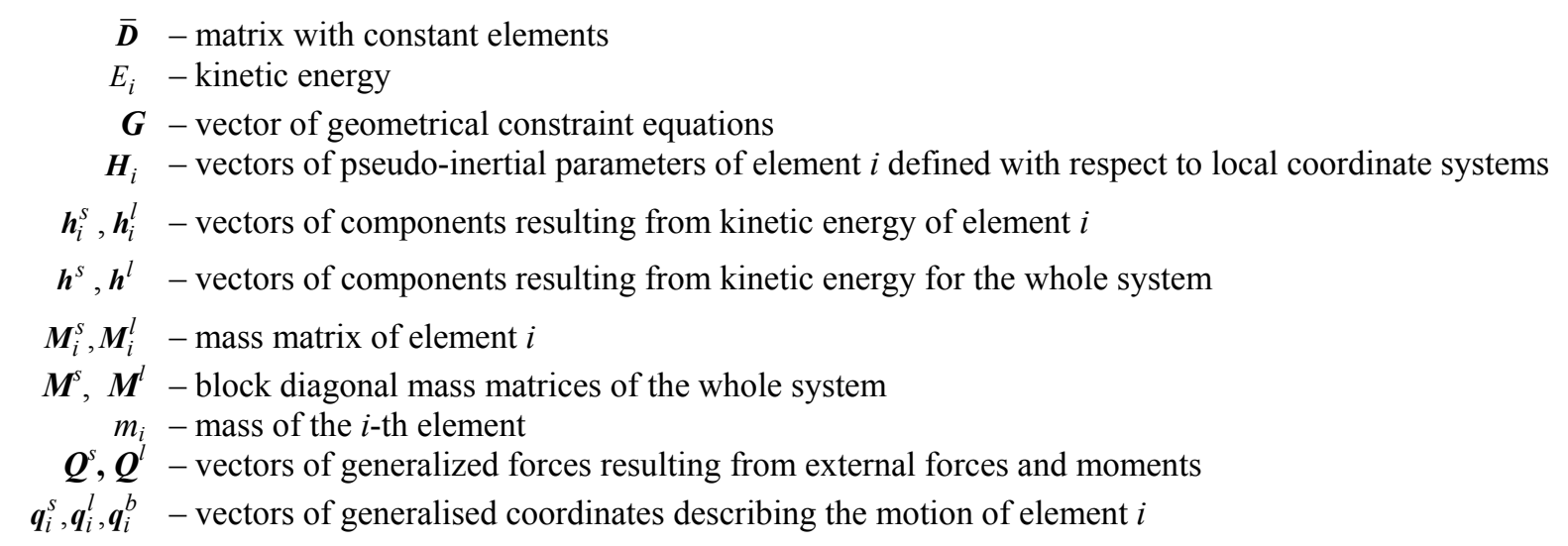




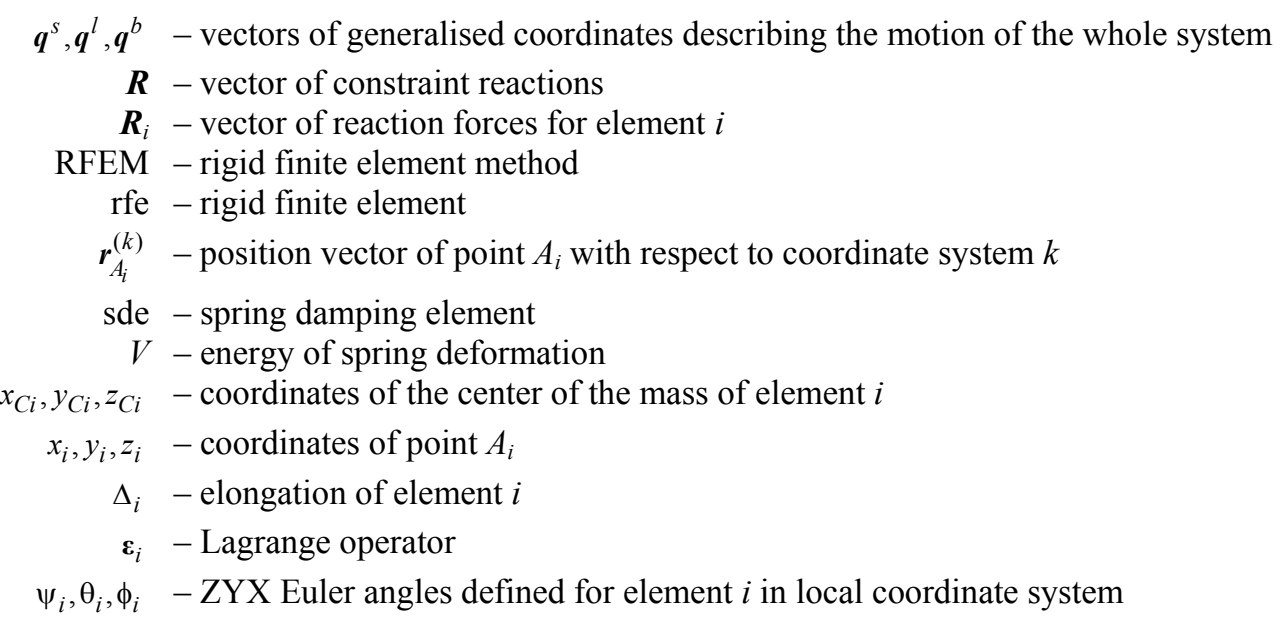

\section{Superscripts}

$b$ - proposed approach in which the longitudinal flexibility is omitted

$l$ - proposed approach in which the longitudinal flexibility is considered

$s$ - classical RFEM

\section{References}

Adamiec-Wójcik I. and Brzozowska L. (2013): Homogenous transformations in dynamics of off-shore slender structures. - Dynamical Systems Theory, Łódź: Press of Łódź University of Technology, pp.307-316.

Adamiec-Wójcik I., Brzozowska L. and Wojciech S. (2013): Modification of the rigid finite element method in modeling dynamics of lines and ropes. - The Archive of Mechanical Engineering, vol.LX, No.3, pp.409-429.

Adamiec-Wójcik I., Wittbrodt E. and Wojciech S. (2012): Rigid finite element in modelling of bending and longitudinal vibrations of ropes. - Int. J. of Applied Mechanics and Engineering, vol.17, No.3, pp.665-67.

Adamiec-Wójcik I. and Wojciech S. (1993): Application of the rigid finite element method in dynamic analysis of plane manipulator. - Mech. Mach. Theory, vol.28, No.3, pp.327-334.

Boer S.E., Aarts R.G.K.M., Meijard J.P., Brouwer D.M. and Jonker J.B. (2014): A nonlinear two-node superelement for use in flexible multibody systems. - Multibody Syst. Dyn., vol.31, No.4, pp.405-431.

Dwivedy S.K. and Eberhard P. (2006): Dynamic analysis of flexible manipulators, a literature review. - Mechanism and Machine Theory, vol.41, pp.749-777.

Kruszewski J. Gawroński W., Wittbrodt E., Najbar F. and Grabowski S. (1975): The Rigid Finite Element Methods. Warsaw: Arkady.

Wittbrodt E., Adamiec-Wójcik I. and Wojciech S. (2006): Dynamics of Flexible Multibody Systems: Rigid Finite Element Method. - Berlin: Springer.

Wittbrodt E., Szczotka M., Maczyński A. and Wojciech S. (2013): Rigid Finite Element Method in Analysis of Dynamics of Offshore Structures. - Berlin: Springer. 